DESY $12-018$

arXiv:1202.0752

February 2012

\title{
Polarized Positrons for the ILC - Update on Simulations
}

\author{
A. Ushakov, O. S. Adeyemi, G. Moortgat-Pick \\ II. Institut für Theoretische Physik, Universität Hamburg \\ F. Staufenbiel, S. Riemann \\ Deutsches Elektronen-Synchrotron DESY, Zeuthen
}

ISSN 0418-9833

NOTKESTRASSE 85 - 22607 HAMBURG 